\title{
Growth Analysis of Watermelon Plants Grown with Mulches and Rowcovers
}

\author{
Nader Soltani ${ }^{1}$, J. LaMar Anderson ${ }^{2}$, and Alvin R. Hamson ${ }^{3}$ \\ Plants, Soils and Biometeorology Department, Utah State University, Logan, UT 84322-4820
}

Additional index words. Citrullus Zunatus, growth analysis, rowcover, mulch, earliness, heat unit, phenological model.

\begin{abstract}
Crimson Sweet' watermelon [Citrullus lanatus (Thunb.) Matsum. \& Nakai] plants were grown with various mulches and rowcovers and analyzed for relative growth rate (RGR), net assimilation rate (NAR), specific leaf area (SLA), leaf area index (LAI), and crop growth rate (CGR). Spunbonded polyester fabric (SB-PF) and perforated polyethylene film (PCP) rowcovers generally showed greater mean RGR, SLA and CGR than spunbonded polypropylene polyamide net (SB-PP), black plus clear combination plastic mulch and black plastic mulch alone. Plants on mulches and under rowcovers showed significant increases in RGR, NAR, and SLA over plants grown in bare soil. Carbon dioxide concentration inside the transplanting mulch holes was nearly twice the ambient CO, concentration. Growth analysis of sampled watermelon plants during early stages of development under various treatments was predictive of crop yield. Plants under SB-PF and PCP rowcovers produced the earliest fruit and the greatest total yield. An asymmetrical curvilinear model for watermelon growth and development based on cardinal temperatures was developed. The model uses hourly averaged temperatures to predict growth and phenological development of 'Crimson Sweet' watermelon plants grown with and without rowcovers. Early vegetative growth correlated well with accumulated heat units. Results indicate a consistent heat unit requirement for the 'Crimson Sweet' watermelon plants to reach first male flower, first female flower and first harvest in uncovered plants and plants under rowcovers. Greater variability was observed in predicting date of first harvest than first bloom.
\end{abstract}

In cool regions of the world, watermelon growth is limited by short growing seasons and low air and soil temperatures, particularly early in the growing season. Low air and soil temperatures, which occur in the spring and early summer, especially during the nights, often delay harvest dates and reduce yield. Rowcovers enhance plant growth and development by modifying air temperature, soil temperature, humidity and light around the covered plants (Mansour, 1989).

Simple models provided by growth analysis techniques describe the productivity of an individual plant or whole canopy of plants grown under rowcovers and help conceptualize carbon partitioning to various plant organs.

Although mulches and rowcovers in various forms have been used for several years, their use has seldom been correlated with environmental factors and phenological development of vegetable crops. Most of this type of research has focused on specific sites and how various mulches and rowcovers affects yield and earliness. Studying the relationship between heat unit accumulation and plant responses can elucidate why rowcovers are so effective in enhancing plant growth and development in one area and less so in other areas (Wolfe et al., 1989).

Many climatic factors effect plant growth and development. Temperature is the most important component of the plant environment since it is an approximate measure of the environment's energy status (Lombard and Richardson, 1979). Temperature data are often incorporated into heat unit models. These models assume that if moisture, nutrients, light and other growth-influencing variables are not limiting, then temperature accounts for most of the variability in plant growth and development.

Received for publication 17 Jan. 1995. Accepted for publication 6 July 1995. This research was supported, in part, by Utah Agricultural Experiment Station, Logan, Utah 84322-4810. Approved as journal paper no. 4619. The use of trade names does not imply endorsement of the products used nor criticism of similar ones not mentioned. The cost of publishing this paper was defrayed in part by the payment of page charges. Under postal regulations, this paper therefore must be hereby marked advertisement solely to indicate this fact.

'Postdoctoral fellow.

${ }^{2}$ Professor.

${ }^{3}$ Emeritus professor.
Wolfe et al. (1989) and Albright et al. (1989) studied the relationship between cumulative degree days and biomass, early and total yield of cucumbers and tomatoes grown under various polyethylene and fabric covers. They found a linear relationship between accumulated degree days with biomass, and total yield of cucumber. Their single regression analysis for cucumber showed $r^{2}$ values between 0.70 to 0.82 .

The predictive accuracy of the degree day models for tomato were not as accurate as with cucumber (Peirce and Crispi, 1989; Wolfe et al., 1989). A negative slope value was associated with the excessive temperatures that often occurred under the rowcovers. The correlation between temperature and yield improved when the model included a high temperature threshold, but accuracy did not improve when light effects and soil temperatures were incorporated (Wolfe et al., 1989).

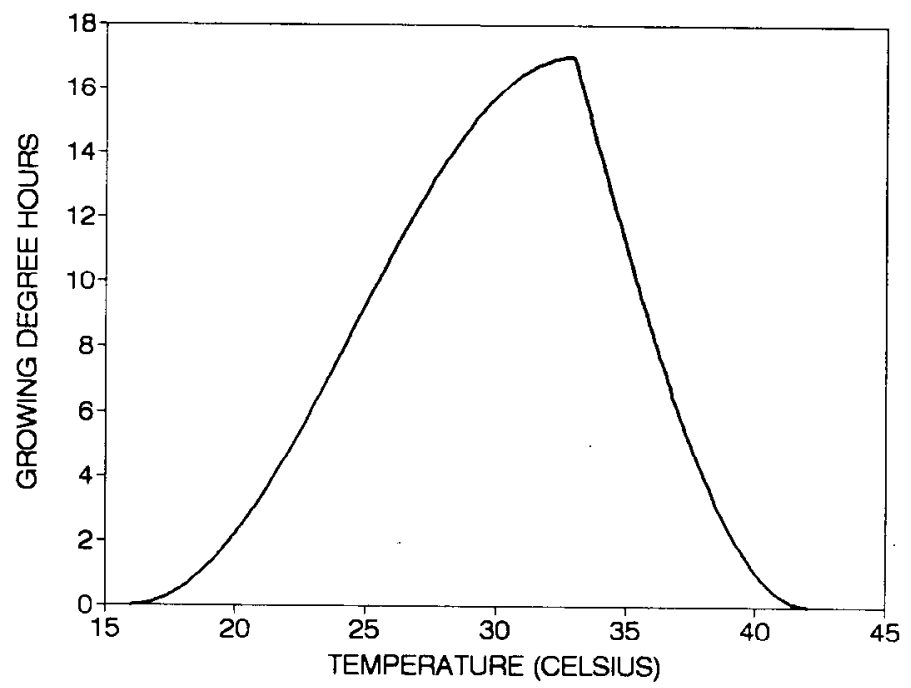

Fig. 1. The computer generated asymmetrical curvilinear temperature model for growth and development of 'Crimson Sweet' watermelon. 
This study uses growth analysis techniques to determine the effects of polyethylene plastic mulches alone and in combination with rowcovers on the growth, earliness and yield of 'Crimson Sweet' watermelon. We also developed an asymmetric curvilinear temperature model based on cardinal temperatures to predict growth and development of watermelon plants grown with various mulches and rowcovers. The model utilizes hourly averaged temperatures which are a more accurate representation of energy status than daily max/min temperatures. Few studies of thermal accumulation under rowcovers have incorporated hourly averaged temperatures into their equations despite the fact that temperatures fluctuate under the rowcovers.

\section{Materials and Methods}

Field studies. Field experiments were conducted at the Greenville Field Station in Logan, Utah, during Summers 1988 to 1991 and at the Farmington Field Station in Kaysville, Utah, during Summer 1991. Watermelon transplants were spaced $1.2 \mathrm{~m}$ apart in the rows, with rows $3 \mathrm{~m}$ apart in a randomized complete block design of four replications. Extra plants per treatment were harvested randomly before transplanting and every week thereafter for growth analysis. Only six plants per plot remained when tunnels were removed. Plots were sprinkler irrigated in 1988 and furrow irrigated under the plastic mulches all other years. Soil moisture was maintained

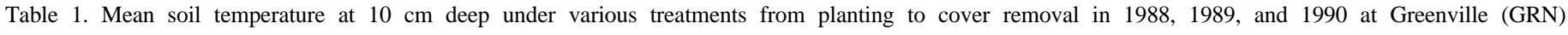
and 1991 at Kaysville (KAYS).

\begin{tabular}{|c|c|c|c|c|c|}
\hline \multicolumn{2}{|c|}{ Treatment $^{2}$} & \multicolumn{4}{|c|}{ Mean soil temp $\left({ }^{\circ} \mathrm{C}\right)$} \\
\hline Mulch & Rowcover & 1988 GRN & 1989 GRN & 1990GRN & 1991 KAYS \\
\hline None & None & $20.0 \mathrm{c}^{y}$ & $18.8 \mathrm{~d}$ & $21.1 \mathrm{~d}$ & $20.7 \mathrm{c}$ \\
\hline BPM & None & $21.9 \mathrm{~b}$ & $20.3 c$ & $23.9 \mathrm{c}$ & $23.1 \mathrm{~b}$ \\
\hline BPM + CPM & None & --- & $22.7 \mathrm{a}$ & $24.7 b$ & $24.9 \mathrm{a}$ \\
\hline CPM & None & --- & --- & $28.5 \mathrm{a}$ & --- \\
\hline BPM & SB-PP & $25.0 \mathrm{a}$ & $21.9 \mathrm{~b}$ & $25.2 \mathrm{~b}$ & $24.2 \mathrm{ab}$ \\
\hline BPM & SB-PF & $24.7 \mathrm{a}$ & $23.0 \mathrm{a}$ & $25.0 \mathrm{~b}$ & $24.6 \mathrm{a}$ \\
\hline BPM & PCP & --- & $22.7 \mathrm{a}$ & $25.4 \mathrm{~b}$ & $24.9 \mathrm{a}$ \\
\hline
\end{tabular}

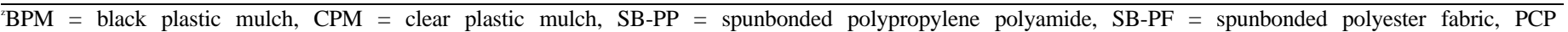
$=$ perforated clear plastic.

${ }^{y}$ Mean separation within columns for each year by Duncan's multiple range test, $P=0.05$.

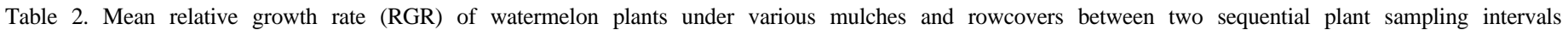
(25 May-8 June and 8-16 June) in 1988, four sampling intervals (24 May-10 June, 10-18 June, 18-25 June, and 25 June-3 July) in 1989, and three sampling intervals (3-20 June, 20-29 June, and 29 June-5 July) in 1990.

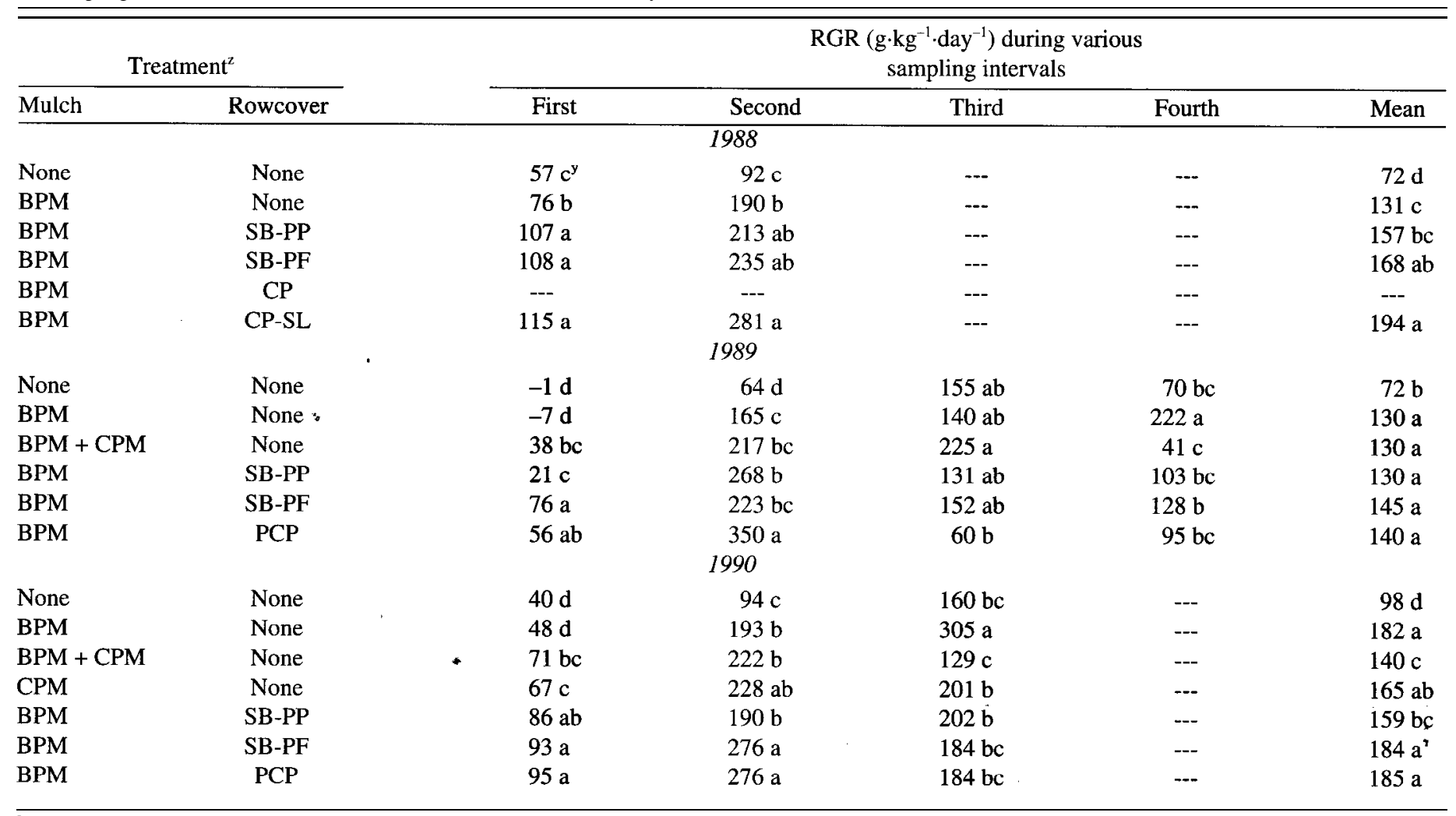

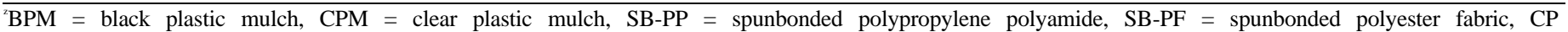
$=$ clear plastic, $\mathrm{CP}-\mathrm{SL}=$ clear plastic slitted, $\mathrm{PCP}=$ perforated clear plastic.

${ }^{y}$ Mean separation within columns for each year by Duncan's multiple range test, $P=0.05$. 
at $50 \%$ field capacity or higher.

In 1988, 'Crimson Sweet' watermelon seedlings were grown to four-true-leaf stage in the greenhouse and were transplanted 24 May. Treatments were 1) bare ground (control); 2) black polyethylene mulch (BPM); and rowcover treatments Were black polyethylene mulch in combination with: 3) spunbonded polypropylenepolyamide net (SB-PP, Agronet); 4) spunbonded polyester fabric (SB-PF, Reemay); 5) clear solid polyethylene film (CP); and 6) clear polyethylene slitted film (CP-SL). Before the mulches were laid on the soil, $85 \mathrm{~g}$ of 11-52-o fertilizer were placed $10 \mathrm{~cm}$ to the side of each plant at 10 to $15 \mathrm{~cm}$ deep. Plants were side dressed with ammonium nitrate after cover removal. Rowcovers were supported by a wire hoop to form tunnels except for SB-PF which was laid over the plants as a floating rowcover. All of the rowcovers were installed 26 May and removed on 22 June.

In 1989, the same general experimental procedures were used. Plants were started in a greenhouse on 4 May and were transplanted to the field on 22 May. Rowcovers were placed over transplants on 24 May. Clear solid and clear slitted polyethylene rowcovers were replaced with 1) black and clear polyethylene mulch combination (BPM $+\mathrm{CPM})$, which were layered $2.5 \mathrm{~cm}$ apart using narrow bricks and 2) a perforated clear polyethylene film (PCP, Vispore) as a rowcover tunnel. All rowcovers were removed on 3 July.

Similar general experimental procedures were followed with one additional mulch treatment of clear polyethylene mulch in 1990. Seedlings were planted on 9 May in the greenhouse and were transplanted on 3 June. Rowcovers were installed on 5 June and removed 4 July.
In 1991, the same procedures and methods as in 1990 were used at Logan and Kaysville, Utah. Seedlings were transplanted to the field 24 May and 6 June in Kaysville and Logan, respectively. Rowcovers were laid 27 May in Kaysville and 7 June in Logan. Covers were removed on 1 July and 9 July in Kaysville and Logan, respectively.

Growth analysis. Plants were harvested weekly in 1988-90 under all treatments until the covers were removed. Four plants per treatment were cut at soil level every week and held on ice until analyzed for stem dry weight, leaf dry weight, number of leaves and leaf area. Leaf areas were measured using a LI-COR leaf area meter. Mean relative growth rate (RGR); net assimilation rate (NAR), specific leaf area (SLA), leaf area index (LAI), and crop growth rate (CGR) were calculated as described by Gardner et al. (1985). A two-way analysis of variance (ANOVA) was used to evaluate each factor for all treatments and sampling intervals.

Environmental measurements. Replicated hourly averaged air and soil temperatures were recorded using Campbell Scientific (Logan, Utah) data loggers $21 \mathrm{X}$ and CR1 0 with AM416 multiplexers. Copper-constantan double-insulated thermocouples (Extension Grade EXTT) were used to sense temperatures $10 \mathrm{~cm}$ below and $10 \mathrm{~cm}$ above ground level. Air temperature thermocouple junctions were shaded in Styrofoam cups. Readings were made by data loggers every $60 \mathrm{sec}$, averaged, and recorded hourly for each treatment from transplanting until last harvest. All thermocouples were calibrated and tested within $\pm 1 \mathrm{C}$ accuracy before installation and after the experiments. Thermocouples that did not maintain $\pm 1 \mathrm{C}$ accuracy were excluded from the data analysis.

Photosynthetically active radiation (PAR) was measured using

Table 3. Mean net assimilation rate (NAR) of watermelon plants under various mulches and rowcovers between two sequential plant sampling intervals (25 May-8 June and 8-16 June) in 1988, four sampling intervals (24 May-10 June 10, 10-18 June, 18-25 June, and 25 June-3 July) in 1989, and three sampling intervals (3-20 June, 20-29 June, and 29 June-5 July) in 1990.

\begin{tabular}{|c|c|c|c|c|c|c|c|}
\hline \multicolumn{2}{|c|}{ Treatment $^{2}$} & & \multicolumn{5}{|c|}{$\begin{array}{l}\text { NAR }\left(\mathrm{g} \cdot \mathrm{m}^{-2} \cdot \mathrm{day}^{-1}\right) \text { during various } \\
\text { sampling intervals }\end{array}$} \\
\hline Mulch & \multicolumn{2}{|l|}{ Rowcover } & First & Second & Third & Fourth & Mean \\
\hline \multicolumn{8}{|c|}{1988} \\
\hline None & None & & $5 b^{y}$ & $13 \mathrm{c}$ & --- & --- & $9 \mathrm{c}$ \\
\hline BPM & None & & $7 b$ & $22 b c$ & --- & --- & $14 \mathrm{~b}$ \\
\hline BPM & SB-PP & & $10 \mathrm{a}$ & $25 \mathrm{~b}$ & --- & --- & $17 \mathrm{~b}$ \\
\hline \multicolumn{8}{|c|}{1989} \\
\hline None & None & & $0 \mathrm{~d}$ & $9 \mathrm{~d}$ & $22 \mathrm{a}$ & $11 \mathrm{bc}$ & $11 \mathrm{~b}$ \\
\hline BPM & None & 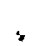 & $-1 \mathrm{~d}$ & $19 c$ & $13 a b$ & $27 \mathrm{a}$ & $14 \mathrm{a}$ \\
\hline $\mathrm{BPM}+\mathrm{CPM}$ & None & & $6 \mathrm{bc}$ & $22 \mathrm{bc}$ & $26 \mathrm{a}$ & $6 c$ & $15 \mathrm{a}$ \\
\hline BPM & SB-PP & & $3 c$ & $26 a b$ & $14 \mathrm{ab}$ & $13 \mathrm{bc}$ & $14 \mathrm{a}$ \\
\hline None & None & & $4 c$ & $11 \mathrm{~d}$ & $18 b c$ & -- & $11 \mathrm{~d}$ \\
\hline $\mathrm{BPM}$ & None & & $4 \mathrm{c}$ & $18 \mathrm{bc}$ & $28 \mathrm{a}$ & --- & $17 \mathrm{a}$ \\
\hline $\mathrm{BPM}+\mathrm{CPM}$ & None & & $.6 a b$ & $20 a b c$ & $12 \mathrm{c}$ & --- & $13 \mathrm{~cd}$ \\
\hline CPM & None & & $5 \mathrm{bc}$ & $21 \mathrm{ab}$ & $23 a b$ & --- & $16 \mathrm{ab}$ \\
\hline BPM & SB-PP & & $6 a b$ & $15 \mathrm{~cd}$ & $18 \mathrm{bc}$ & --- & $13 \mathrm{bcd}$ \\
\hline $\mathrm{BPM}$ & SB-PF & & $7 \mathrm{a}$ & $22 a b$ & $15 b c$ & -- & $15 a b c$ \\
\hline BPM & PCP & & $8 \mathrm{a}$ & $23 \mathrm{a}$ & $16 \mathrm{bc}$ & --- & $15 \mathrm{abc}$ \\
\hline
\end{tabular}

$\overline{\mathrm{BPM}}=$ black plastic mulch, $\mathrm{CPM}=$ clear plastic mulch, $\mathrm{SB}-\mathrm{PP}=$ spunbonded polypropylene polyamide, $\mathrm{SB}-\mathrm{PF}=$ spunbonded polyester fabric, $\mathrm{CP}$ $=$ clear plastic, $\mathrm{CP}-\mathrm{SL}=$ clear plastic slitted, $\mathrm{PCP}=$ perforated clear plastic.

'Mean separation within columns for each year by Duncan's multiple range test, $P=0.05$. 
a LI-COR (model 190SB) quantum sensor at plant level under the covered and uncovered plants on a clear sunny day at noon in 1990. Carbon dioxide was measured with a Beckman infrared gas analyzer (IRGA) under both calm and windy weather conditions when photosynthetic activity was at its peak under the rowcovers. Air samples were drawn using 5-ml syringes $10 \mathrm{~cm}$ above ground level from all treatments and from the holes where plants came through the plastic mulches. Air samples were injected into the IRGA and $\mathrm{CO}_{2}$ concentrations were charted.

Phenological measurements. Dates of the following phenological observations were recorded for all treatments: 1) first male blossom, 2) first female blossom, 3) first fruit set, and 4) first harvest. Four fruits per treatment were selected randomly in two representative blocks at anthesis. Fruit enlargement (circumference) was then measured weekly until maturity and harvest.

Model development. Leopold (1964) stated that a temperature response curve for plant growth should follow the shape of an enzyme response curve, rising rapidly in the lower temperature range, remaining constant in the intermediate range, and then falling at higher temperatures. Incorporation of a nonlinear response to temperature is one way to improve thermal models (Wang, 1960). A sharp decrease in corn (Levitt, 1980) and tomato (Went, 1945) growth rates occurs when plant temperatures rise above the optimum temperature.

A recent asymmetric model (Richardson and Leonard, 1981), uses two modifications of the thermal model concept. First, it incorporates an upper threshold or critical temperature and second, it introduces a nonlinear growth response to temperature. This model uses hourly temperatures which are accurate representa- tions of thermal units received by plants during growth. As the response of a plant species does not follow a true cosine curve (Richardson and Leonard, 1981), the response rate to increasing temperatures above the optimum (Eq. [2]) is different from that below the optimum (Eq. [1]),

$$
\begin{aligned}
& \mathrm{GDH}=\mathrm{FA} / 2\{1+\cos [\pi+\pi(\mathrm{TH}-\mathrm{TB} / \mathrm{TU}-\mathrm{TB})]\} \\
& \text { for } \mathrm{TH} \text { less than or equal to } \mathrm{TU}
\end{aligned}
$$

$\mathrm{GDH}=\mathrm{FA}\{1+\cos [\pi / 2+\pi / 2(\mathrm{TH}-\mathrm{TU} / \mathrm{TC}-\mathrm{TU})]\}$

for $\mathrm{TH}$ greater than or equal to $\mathrm{TU}$

where GDH = accumulation of growing degree hours during an hour, $\mathrm{TH}=$ hourly temperature, $\mathrm{TB}=$ base temperature, $\mathrm{TU}=$ the optimum temperature, $\mathrm{TC}=$ the critical temperature, $\mathrm{A}=\mathrm{TU}-\mathrm{TB}$ (the amplitude of the growth curve), and $\mathrm{F}=$ a stress factor (soil moisture stress, stress from disease, insect damage, or lack of nutrients)

If the value of $\mathrm{TH}$ is less than or equal to TU, Eq. [1] should be used in accumulation of GDH; if TH is greater than or equal to TU, Eq. [2] should be used. Anderson and Richardson (1982) also reported a marked improvement in tomato bloom predictability when both equations were used to calculate the GDH accumulation.

The watermelon model developed in this study is based upon the equations developed by Richardson and Leonard (1981) and is shown in the growth curve in Fig. 1. Cardinal temperatures used in the model are $\mathrm{TB}=16 ; \mathrm{TU}=33$; $\mathrm{TC}=42$ (Buttrose and Sedgley, 1978; Lorenz and Maynard, 1980; Sedgley and Buttrose, 1978).

Table 4. Mean specific leaf area (SLA) of watermelon plants under various mulches and rowcovers between two sequential plant sampling intervals (25 May-8 June and 8-16 June) in 1988, four sampling intervals (24 May-10 June, 10-18 June, 18-25 June and 25 June-3 July) in 1989, and three

\begin{tabular}{|c|c|c|c|c|c|c|}
\hline \multicolumn{2}{|c|}{ Treatment $^{z}$} & \multicolumn{5}{|c|}{$\begin{array}{c}\text { SLA }\left(\mathrm{m}^{2} \cdot \mathrm{kg}^{-1}\right) \text { during various } \\
\text { sampling intervals }\end{array}$} \\
\hline Mulch & Rowcover & First & Second & Third & Fourth & Mean \\
\hline \multicolumn{7}{|c|}{1988} \\
\hline None & None & $16.8 \mathrm{a}^{\mathrm{y}}$ & $10.4 \mathrm{~b}$ & --- & --- & $13.6 \mathrm{a}$ \\
\hline BPM & None & $16.8 \mathrm{a}$ & $12.2 \mathrm{a}$ & --- & --- & $14.5 \mathrm{a}$ \\
\hline BPM & SB-PP & $17.1 \mathrm{a}$ & $11.7 \mathrm{a}$ & --- & --- & $14.4 \mathrm{a}$ \\
\hline BPM & SB-PF & $17.2 \mathrm{a}$ & $11.9 \mathrm{a}$ & --- & --- & $14.6 \mathrm{a}$ \\
\hline BPM & $\mathrm{CP}$ & --- & --- & -- & --- & --- \\
\hline BPM & CP-SL & $16.7 \mathrm{a}$ & $\begin{array}{l}11.5 \mathrm{ab} \\
1989\end{array}$ & --- & --- & $14.1 \mathrm{a}$ \\
\hline None & None & $8.2 \mathrm{~d}$ & $10.4 \mathrm{e}$ & $10.5 \mathrm{~d}$ & $8.9 \mathrm{~d}$ & $9.5 \mathrm{~d}$ \\
\hline BPM & None & $8.7 \mathrm{~cd}$ & $11.7 \mathrm{de}$ & $14.6 \mathrm{ab}$ & $12.5 \mathrm{a}$ & $11.9 \mathrm{bc}$ \\
\hline $\mathrm{BPM}+\mathrm{CPM}$ & None & $9.4 b c$ & $12.7 \mathrm{~cd}$ & $13.0 \mathrm{c}$ & $9.7 \mathrm{c}$ & $11.2 \mathrm{c}$ \\
\hline BPM & SB-PP & $10.1 \mathrm{ab}$ & $13.9 \mathrm{bc}$ & $13.8 \mathrm{bc}$ & $11.1 \mathrm{~b}$ & $12.2 \mathrm{ab}$ \\
\hline BPM & SB-PF & $11.0 \mathrm{a}$ & $15.8 \mathrm{a}$ & $15.4 \mathrm{a}$ & $11.7 \mathrm{~b}$ & $13.5 \mathrm{a}$ \\
\hline BPM & PCP & $10.1 \mathrm{ab}$ & $\begin{array}{l}14.6 \mathrm{ab} \\
1990\end{array}$ & $14.5 \mathrm{ab}$ & $11.4 \mathrm{~b}$ & $12.7 \mathrm{ab}$ \\
\hline None & None & $20.8 \mathrm{a}$ & $13.4 \mathrm{e}$ & $12.1 \mathrm{e}$ & --- & $15.4 \mathrm{~d}$ \\
\hline BPM & None & $20.8 \mathrm{a}$ & $15.0 \mathrm{bcd}$ & $15.8 \mathrm{bc}$ & --- & $17.2 \mathrm{bc}$ \\
\hline $\mathrm{BPM}+\mathrm{CPM}$ & None & $20.5 \mathrm{a}$ & $14.7 \mathrm{cde}$ & $15.0 \mathrm{~cd}$ & --- & $16.7 \mathrm{c}$ \\
\hline CPM & None & $20.8 \mathrm{a}$ & $14.5 \mathrm{de}$ & $13.7 \mathrm{de}$ & --- & $16.3 \mathrm{~cd}$ \\
\hline BPM & SB-PP & $21.2 \mathrm{a}$ & $16.3 \mathrm{ab}$ & $16.1 \mathrm{bc}$ & --- & $17.9 \mathrm{ab}$ \\
\hline BPM & SB-PF & $21.0 \mathrm{a}$ & $16.9 \mathrm{a}$ & $18.5 \mathrm{a}$ & ..- & $18.8 \mathrm{a}^{\prime}$ \\
\hline BPM & PCP & $20.7 \mathrm{a}$ & $16.1 \mathrm{abc}$ & $17.4 \mathrm{ab}$ & --- & $18.1 \mathrm{ab}$ \\
\hline
\end{tabular}
sampling intervals (3-20 June, 20-29 June, and 29 June-5 July) in 1990.

$\overline{\mathrm{BPM}}=$ black plastic mulch, $\mathrm{CPM}=$ clear plastic mulch, $\mathrm{SB}-\mathrm{PP}=$ spunbonded polypropylene polyamide, $\mathrm{SB}-\mathrm{PF}=\mathrm{spunbonded}$ polyester fabric, $\mathrm{CP}$ = clear plastic, $\mathrm{CP}-\mathrm{SL}=$ clear plastic slitted, $\mathrm{PCP}=$ perforated clear plastic .

'Mean separation within columns for each year by Duncan's multiple range test, $P=0.05$. 
Due to the cosine curve's asymptotical approach to base (TB) and critical (TC) temperatures, inaccuracies in the approximate base and critical temperatures result in less error than inaccuracies in approximate value of the optimum temperature.

\section{Results and Discussion}

In 1988, excessive heat build up under the clear plastic rowcover treatment resulted in severe injury to watermelon plants and therefore this treatment was abandoned. Plants under clear slitted treatments also had to be further ventilated at later growth stages to avoid plant desiccation due to high temperatures.

Light intensity at the plant level under rowcovers was lower than for uncovered plants. PCP and SB-PP rowcovers transmitted about $80 \%$ of photosynthetic photon flux (PPF) while the SB-PF rowcover transmitted about $70 \%$ of PPF. However, as Wells and Loy (1985) reported, the reduction in transmittance should not limit plant growth in full or partial sun, because even under these conditions PPF often exceeds the light saturation point of the plants.

Carbon dioxide concentration inside the mulch transplanting hole $\left(600 \mu \mathrm{l} \cdot\right.$ liter $\left.^{-1}\right)$ was significantly greater than ambient $\mathrm{CO}_{2}$ concentration $\left(\mu \mathrm{l} \cdot\right.$ liter $\left.^{-1}\right)$. However, samples taken a few $\mathrm{cm}$ away from the hole at foliage level showed no significant differences in $\mathrm{CO}_{2}$ concentration from ambient suggesting that $\mathrm{CO}_{2}$ gradients above these holes were dissipated rapidly by air movement. The elevated $\mathrm{CO}_{2}$ concentration inside the holes may have been benefi- cial during early stages of seedling growth on a calm day, but was unlikely to be of significance under the mulches and rowcovers mainly due to the wind prevalent at both locations.

Mean soil temperatures under all mulches and rowcover treatments were significantly higher than mean soil temperature under bare ground (Table 1). In 1990 mean soil temperatures under clear mulches averaged 7.4C higher than bare ground soil temperature and about $3 \mathrm{C}$ higher than all other treatments. Clear plastic transmits most of the incoming solar radiation to the soil, and then retards the loss of soil heat. However, black mulch re-radiates most of the solar energy as sensible heat to the air or to the soil (Wolfe et al., 1989). Black polyethylene mulch increased mean soil temperature over that of bare ground by $1.9,1.5,2.8$, and $2.4 \mathrm{C}$ during 1988, 1989, 1990, and 1991, respectively. Theseresults are generally similar to those found by other investigators (Hemphill and Crabtree, 1988; Hemphill and Mansour, 1986). In 1988, SB$\mathrm{PP}$ and SB-PF rowcovers increased mean soil temperature more than $2.5 \mathrm{C}$ over that of black polyethylene mulch alone. Rowcovers plus BPM increased mean soil temperature 1.1 to $2.7 \mathrm{C}$ more than BPM alone during 1989-90. The combination of black and clear plastic mulch increased mean soiltemperature over that of BPM by 2.4, 0.8, and 1.8C during 1989, 1990, and 1991, respectively.

In 1988, mulch and rowcovers enhanced relative growth rate, especially during the first sampling period when the growth of uncovered plants (BPM and bare ground) was lower than of rowcover plants (Table 2). There was a steady increased mean RGR early in the season when temperatures were not excessive.

Table 5. Mean leaf area index (LAI) of watermelon plants under various mulches and rowcovers between two sequential plant sampling intervals (25 May-8 June and 8-16 June) in 1988, four sampling intervals (May 24-June 10, 10-18 June, 18-25 June, and June 25-July 3) in 1989, and three sampling intervals (3-20 June, 20-29 June, and 29 June-5 July) in 1990.

\begin{tabular}{|c|c|c|c|c|c|c|c|}
\hline \multicolumn{3}{|c|}{ Treatment $^{2}$} & \multicolumn{5}{|c|}{$\begin{array}{c}\mathrm{LAI}\left(\mathrm{m}^{2} \text { leaf } / \mathrm{m}^{2} \text { ground }\right) \text { during } \\
\text { various sampling intervals } \\
(\mathrm{LAI} \times 1000)\end{array}$} \\
\hline Mulch & Rowcover & & First & Second & Third & Fourth & Mean \\
\hline & & & & 1988 & & & \\
\hline None & None & & $1.62 b^{y}$ & $2.47 \mathrm{~d}$ & --- & --- & $2.04 \mathrm{~d}$ \\
\hline BPM & None & & $1.99 \mathrm{~b}$ & $8.13 c$ & --- & --- & $5.06 \mathrm{c}$ \\
\hline BPM & SB-PP & & $2.86 \mathrm{a}$ & $14.04 \mathrm{~b}$ & --- & --- & $8.45 b$ \\
\hline BPM & SB-PF & & $2.93 \mathrm{a}$ & $16.78 b$ & --- & --- & $9.86 \mathrm{~b}$ \\
\hline BPM & $\mathrm{CP}$ & & --- & --- & --- & --- & --- \\
\hline BPM & CP-SL & & $3.14 \mathrm{a}$ & $\begin{array}{l}23.34 \mathrm{a} \\
1989\end{array}$ & --- & --- & $13.24 \mathrm{a}$ \\
\hline None & None & & $0.42 \mathrm{e}$ & $0.91 \mathrm{~d}$ & $2.10 \mathrm{~d}$ & $4.18 c$ & $1.90 \mathrm{~d}$ \\
\hline BPM & None & $\therefore$ & $0.47 \mathrm{de}$ & $2.10 \mathrm{~d}$ & $6.60 \mathrm{~d}$ & $23.25 \mathrm{~b}$ & $8.11 \mathrm{c}$ \\
\hline $\mathrm{BPM}+\mathrm{CPM}$ & None & & $0.93 \mathrm{c}$ & $6.71 \mathrm{c}$ & $26.18 \mathrm{c}$ & $38.66 \mathrm{~b}$ & $18.12 b$ \\
\hline BPM & SB-PP & & $0.76 \mathrm{~cd}$ & $7.43 \mathrm{c}$ & $19.85 \mathrm{c}$ & $37.55 \mathrm{~b}$ & $16.40 \mathrm{~b}$ \\
\hline BPM & SB-PF & & $1.79 \mathrm{a}$ & $15.04 \mathrm{~b}$ & $38.07 \mathrm{~b}$ & $76.32 \mathrm{a}$ & $32.81 \mathrm{a}$ \\
\hline BPM & PCP & & $1.34 \mathrm{~b}$ & $\begin{array}{l}24.95 \mathrm{a} \\
1990\end{array}$ & $48.14 \mathrm{a}$ & $70.17 \mathrm{a}$ & $36.15 \mathrm{a}$ \\
\hline None & None & & $0.26 \mathrm{~d}$ & $2.51 \mathrm{c}$ & $10.66 \mathrm{~b}$ & --- & $4.47 \mathrm{~b}$ \\
\hline BPM & None & & $1.10 \mathrm{c}$ & $2.75 \mathrm{c}$ & $12.32 \mathrm{~b}$ & --- & $5.39 \mathrm{~b}$ \\
\hline $\mathrm{BPM}+\mathrm{CPM}$ & None & & - $2.10 \mathrm{ab}$ & $21.81 \mathrm{a}$ & $77.86 \mathrm{a}$ & --- & $33.92 \mathrm{a}$ \\
\hline CPM & None & & $2.18 \mathrm{a}$ & $21.56 \mathrm{a}$ & $75.73 \mathrm{a}$ & --- & $33.16 \mathrm{a}$ \\
\hline BPM & SB-PP & & $1.51 \mathrm{bc}$ & $7.29 \mathrm{bc}$ & $32.51 \mathrm{~b}$ & $\ldots$ & $13.77 \mathrm{~b}$ \\
\hline BPM & SB-PF & & $2.17 \mathrm{a}$ & $15.17 \mathrm{ab}$ & $49.62 \mathrm{ab}$ & --- & $22.32 \mathrm{ab}$ \\
\hline BPM & PCP & & $1.96 \mathrm{ab}$ & $15.11 \mathrm{ab}$ & $43.03 \mathrm{ab}$ & -.- & $20.04 a b$ \\
\hline
\end{tabular}

$\overline{{ }^{2} \mathrm{BPM}}=$ black plastic mulch, $\mathrm{CPM}=$ clear plastic mulch, $\mathrm{SB}-\mathrm{PP}=$ spunbonded polypropylene polyamide, $\mathrm{SB}-\mathrm{PF}=$ spunbonded polyester fabric, $\mathrm{CP}$ $=$ clear plastic, $\mathrm{CP}-\mathrm{SL}=$ clear plastic slitted, $\mathrm{PCP}=$ perforated clear plastic.

'Mean separation within columns for each year by Duncan's multiple range test, $P=0.05$. 
Table 6. Mean crop growth rate (CGR) of watermelon plants under various mulches and rowcovers between two sequential plant sampling intervals (25 May-8 June and 8-16 June) in 1988, four sampling intervals (24 May-10 June, 10-18 June, 18-25 June, and 25 June-3 July) in 1989, and three sampling intervals (3-20 June, 20-29 June, and 29 June-5 July) in 1990.

\begin{tabular}{|c|c|c|c|c|c|c|}
\hline \multicolumn{2}{|c|}{ Treatment $^{x}$} & - & \multicolumn{3}{|c|}{$\begin{array}{c}\text { CGR }\left(\mathrm{g} \cdot \mathrm{m}^{-2} \cdot \mathrm{day}^{-1}\right) \text { during various } \\
\text { sampling intervals } \\
(\mathrm{CGR} \times 1000)\end{array}$} & Mean \\
\hline \multicolumn{7}{|c|}{1988} \\
\hline None & None & $1.9 b^{y}$ & $32.1 \mathrm{c}$ & --- & --- & $17 \mathrm{c}$ \\
\hline BPM & None & $9.8 \mathrm{~b}$ & $142.3 \mathrm{c}$ & --- & --- & $76 c$ \\
\hline BPM & $\mathrm{CP}$ & --- & --- & --- & --- & -.. \\
\hline BPM & CP-SL & $31.5 \mathrm{a}$ & $\begin{array}{l}586.1 \mathrm{a} \\
1989\end{array}$ & --- &.-- & $309 \mathrm{a}$ \\
\hline None & None & $-0.5 \mathrm{~d}$ & $7.8 \mathrm{e}$ & $44.3 \mathrm{~b}$ & $44.1 \mathrm{~d}$ & $24 c$ \\
\hline BPM & None & $-0.3 \mathrm{~d}$ & $29.7 \mathrm{de}$ & $81.0 \mathrm{~b}$ & $556.7 \mathrm{bc}$ & $167 \mathrm{~b}$. \\
\hline $\mathrm{BPM}+\mathrm{CPM}$ & None & $5.2 \mathrm{c}$ & $111.4 \mathrm{~cd}$ & $596.6 \mathrm{a}$ & $185.5 \mathrm{~cd}$ & $225 \mathrm{~b}$ \\
\hline None & None & $3.6 \mathrm{~d}$ & $18.5 \mathrm{c}$ & $79.0 \mathrm{~d}$ & --- & $34 \mathrm{~d}$ \\
\hline BPM & None & $4.9 \mathrm{~cd}$ & $76.6 \mathrm{bc}$ & $724.4 \mathrm{~b}$ & --- & $269 \mathrm{bc}$ \\
\hline $\mathrm{BPM}+\mathrm{CPM}$ & None & $9.0 \mathrm{bc}$ & $151.6 \mathrm{~b}$ & $304.9 \mathrm{~cd}$ & --- & $155 \mathrm{c}$ \\
\hline CPM & None & $8.2 \mathrm{bcd}$ & $154.0 \mathrm{~b}$ & $683.1 \mathrm{~b}$ & --- & $282 \mathrm{~b}$ \\
\hline BPM & SB-PP & $12.5 \mathrm{ab}$ & $141.2 \mathrm{~b}$ & $612.4 b c$ & --- & $255 \mathrm{bc}$ \\
\hline BPM & SB-PF & $14.7 \mathrm{a}$ & $389.2 \mathrm{a}$ & $1250.0 \mathrm{a}$ & --- & $551 \mathrm{a}$ \\
\hline BPM & PCP & $16.1 \mathrm{a}$ & $396.0 \mathrm{a}$ & $1303.3 \mathrm{a}$ & --- & $572 a$ \\
\hline
\end{tabular}

In 1989, ambient temperatures were low during the first sampling interval (24 May-10 June) and RGR was negative by plants on BPM and bare ground treatment but was positive by plants under all other treatments. During the second sampling interval (10-18 June) mean RGR under PCP rowcover was higher than other treatments. All mulch treatments had significantly higher mean RGR than bare ground treatment. However, during the third sampling interval (18-25 June) mean RGR of rowcover plants was generally less than that of noncovered plants mainly due to excessive temperatures occurring under the rowcovers during that period. The mean RGR of plants on BPM + CPM combination mulch was nearly 3 times greater that for than PCP rowcover. During the fourth sampling interval (25 June-3 July) mean RGR was again lower under PCP cover, indicating that the growth rate of plants was depressed by high temperatures. Mean RGR of BPM uncovered plants was significantly higher than plants in other treatments during this sampling interval. Similar temperature effects have been reported by other researchers (Wells and Loy,

In 1990, mean RGR of plants under rowcovers was significantly higher during the first (3-20 June) and second (20-29 June) sampling intervals, but was lower than uncovered BPM plants in the third sampling interval (29 June-5 July). There were no significant differences in mean RGR between rowcover treatments and bare or CPM treatments suggesting that removal of covers before the third interval might have enhanced the mean RGR.
In 1989, very low ambient temperatures resulted in virtually no net assimilation rate (NAR) under BPM and on bare ground during the first sampling intervals (Table 3). However, mean NAR was greater with BPM + CPM and all of the rowcover treatments had a greater mean NAR. The NAR with the SB-PF rowcover treatment was 10 times greater than on bare ground or BPM treatment during the first sampling interval. In the second interval, plants under PCP and SB-PP continued to have a greater mean NAR. In the third sampling interval when temperatures were higher, the NAR of covered plants declined sharply while the NAR of plants on bare ground was the highest. At this stage, the NAR of plants in bare ground was nearly 3 times greater than plants under PCP rowcovers. During the fourth sampling interval the mean NAR in all treatments was generally similar, perhaps due to fluctuating ambient temperatures.

In 1988 , there were no significant differences in specific leaf area (SLA) for the two combined sampling intervals (Table 4). However, in the second sampling, the SLA of plants bare ground was significantly lower than the SLA of plants grown with BPM alone or in combination with SB-PP or SB-PF rowcovers.

In 1989, the SLA of all mulched and covered plants exceeded that of plants on bare ground. Means of overall sampling intervals indicated that SB-PF cover treatments induced the greatest SLA, probably due to faster growth rate of plants.

Examining mean leaf area index (LAI) in 1988 indicated higher mean LAI values under all rowcover treatments than the BPM treatment which, in turn, had a higher LAI value than the bare ground treatment (Table 5). In 1989, the overall mean crop LAI 
Table 7. Early and total yield of 'Crimson Sweet' watermelons under various treatments in 1989-91 at Greenville and in 1991 at Kaysville.

\begin{tabular}{|c|c|c|c|c|c|c|c|c|c|}
\hline & & \multicolumn{8}{|c|}{ Yield (tons/ha) } \\
\hline \multicolumn{2}{|c|}{ Treatment $^{2}$} & \multicolumn{2}{|c|}{1989 Greenville } & \multicolumn{2}{|c|}{1990 Greenville } & \multicolumn{2}{|c|}{1991 Greenville } & \multicolumn{2}{|c|}{1991 Kaysville } \\
\hline Mulch & Rowcover & Early $^{y}$ & Total & Early & Total & Early & Total & Early & Total \\
\hline BPM & None & $0.0 \mathrm{c}$ & $51.1 \mathrm{~b}$ & $11.7 \mathrm{~b}$ & $55.1 \mathrm{c}$ & $8.2 \mathrm{~b}$ & $41.0 \mathrm{~b}$ & $14.7 \mathrm{~b}$ & $56.2 \mathrm{~b}$ \\
\hline $\mathrm{BPM}+\mathrm{CPM}$ & None & $7.0 \mathrm{~b}$ & $51.9 \mathrm{~b}$ & $12.3 \mathrm{~b}$ & $57.2 \mathrm{c}$ & $9.0 \mathrm{~b}$ & $45.7 \mathrm{ab}$ & $17.9 \mathrm{ab}$ & $52.3 \mathrm{~b}$ \\
\hline CPM & None & -..- & --- & $20.6 \mathrm{ab}$ & $55.6 \mathrm{c}$ & $8.7 \mathrm{~b}$ & $47.1 \mathrm{ab}$ & -.-- & ---- \\
\hline BPM & PCP & $14.0 \mathrm{a}$ & $62.8 \mathrm{a}$ & $28.8 \mathrm{a}$ & $68.7 \mathrm{ab}$ & $11.9 \mathrm{ab}$ & $53.5 \mathrm{a}$ & $22.3 \mathrm{a}$ & $59.9 \mathrm{~b}$ \\
\hline
\end{tabular}

$\overline{{ }^{\mathrm{z}} \mathrm{BPM}}=$ black plastic mulch, $\mathrm{CPM}=$ clear plastic mulch, $\overline{\mathrm{SB}}-\mathrm{PP}=$ spunbonded polypropylene polyamide, $\overline{\mathrm{SB}-\mathrm{PF}}=\mathrm{spunbonded}$ polyester fabric, $\mathrm{PCP}$ $=$ perforated clear plastic.

'Early yield = total yield of watermelon harvested by second harvest week.

'Mean separation within columns for each date by Duncan's multiple range test, $P=0.05$.

Table 8. Accumulation of mean growing degree hours $( \pm \mathrm{SD})$ from transplanting to various phenological stages for 'Crimson Sweet' watermelon grown under various treatments during 1988-91.

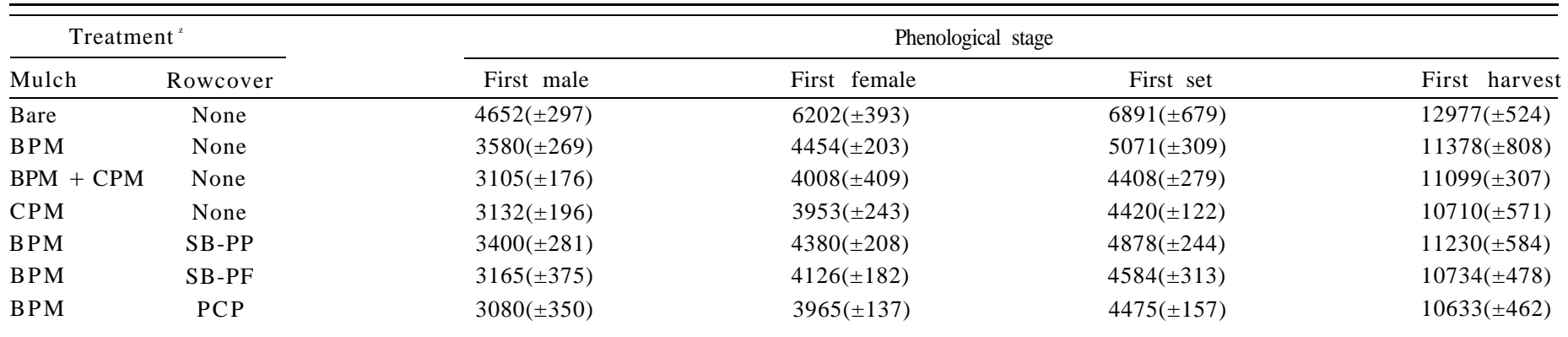

${ }^{2} \mathrm{BPM}=$ black plastic mulch, $\mathrm{CPM}=$ clear plastic mulch, SB-PP = spunbonded polypropylene polyamide, $\mathrm{SB}-\mathrm{PF}=\mathrm{spunbonded}$ polyester fabric and $\mathrm{PCP}=$ perforated clear plastic.

was similar to that in 1988. The mean LAI of PCP and SB-PF covered plants exceeded that of SB-PP or BPM + CPM plants which, in turn, exceeded that of BPM and bare ground plants. In 1990, the mean LAI of plants in BPM + CPM and CPM mulches was higher than of plants in BPM and bare ground.

In 1988, rowcovers substantially increased mean crop growth rate (CGR) (Table 6). Plant canopies under CP-SL cover accumulated dry matter per unit land area nearly 4 times faster than BPM plant canopy and nearly 18 times faster than on bare ground. In 1989, plants on bare and BMP treatments initially had a negative mean CGR mainly due to cold ambient temperatures while during the same time period mean CGR increased for covered plants, especially SB-PF.

Total yield of watermelon fruit harvested by the second week was considered as early yield. hi 1989, early yield was highest under the SB-PF and PCP rowcover treatments followed by SB-PP rowcover and BPM + CPM combination mulch which, in turn, were greater than BPM alone or bare soil treatments (Table 7). Total yields under PCP and SB-PF rowcovers were $44 \%$ greater than on bare ground. Black polyethylene mulch and BPM + CPM combination mulch treatments increased the total yield by about $33 \%$ over the bare ground treatment. These results are consistent with early leaf area development and growth analysis as shown in Tables 2-6. Plants under rowcovers having the highest growth rate and leaf area development presumably supplied a greater amount of assimilates during fruit sizing and maturation, therefore increased earliness and yield.

In 1990, yield patterns were generally similar to those in 1989. Early yield of plants under PCP and SB-PF rowcovers was about $58 \%$ greater than BPM (Table 7). Total yield was also greatest
Table 9. The correlation $\left(r^{2}\right)$ between accumulated growing degree hours and mean leaf numbers, leaf area, leaf dry weight (LDW) and stem dry weight (SDW) of 'Crimson Sweet' watermelon plants under various treatments from transplanting to cover removal during 1988-90. All correlations were highly significant $(\mathrm{a}=0.0000)$.

\begin{tabular}{lcccc}
\hline \hline & \multicolumn{4}{c}{ GDH correlation $\left(r^{2}\right)$} \\
\cline { 2 - 5 } Treatment $^{2}$ & Leaf no. & Leaf area & LDW & SDW \\
\hline Bare & 0.88 & 0.86 & 0.85 & 0.75 \\
BPM & 0.90 & 0.78 & 0.84 & 0.80 \\
BPM + CPM & 0.89 & 0.78 & 0.73 & 0.71 \\
BPM + SB-PP & 0.93 & 0.83 & 0.91 & 0.88 \\
BPM + SB-PF & 0.92 & 0.76 & 0.90 & 0.87 \\
BPM + PCP & 0.94 & 0.70 & 0.92 & 0.93
\end{tabular}

${ }^{2} \mathrm{BPM}=$ black plastic mulch, $\mathrm{CPM}=$ clear plastic mulch, $\mathrm{SB}-\mathrm{PP}=$ spunbonded polypropylene polyamide; $\mathrm{SB}-\mathrm{PF}=$ spunbonded polyester fabric and $\mathrm{PCP}=$ perforated clear plastic.

under the SB-PF and PCP rowcover treatments. Compared to bare ground, rowcovers increased total yield $32 \%$ to $42 \%$, while BPM, $\mathrm{CPM}$ and BPM + CPM mulches increased total yield by $21 \%, 21 \%$, and $24 \%$ over plants on bare ground.

In 1991, yields at both locations were similar to those of previous years. First harvest date of mulch and rowcover plants was advanced 13 and 20 days at Greenville and Kaysville, respectively. Early fruit yield with PCP and SB-PF treatments exceeded those of all mulches, which in turn exceeded yields on bare ground (Table 7). Similar findings have been reported by other researchers working with watermelons and muskmelons (Mansour, 1989; Marr et al., 1991; Miller, 1989; Wilson et al., 1987). 


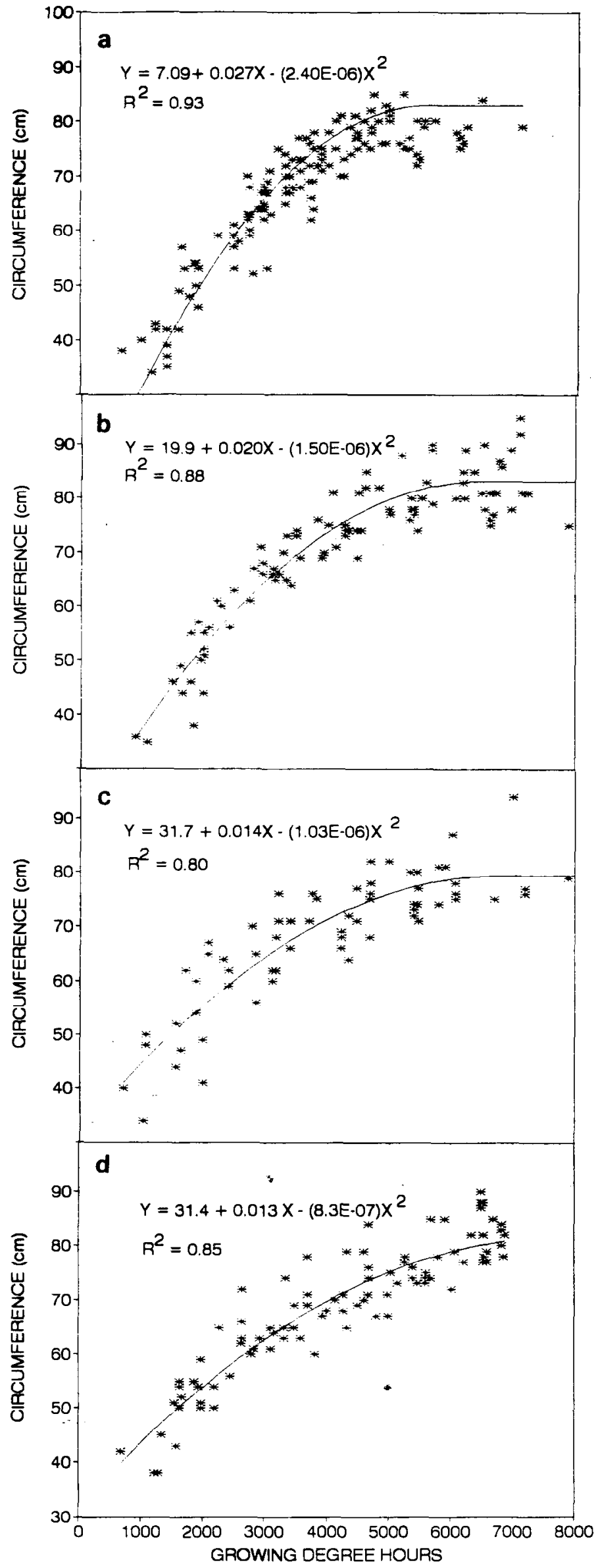

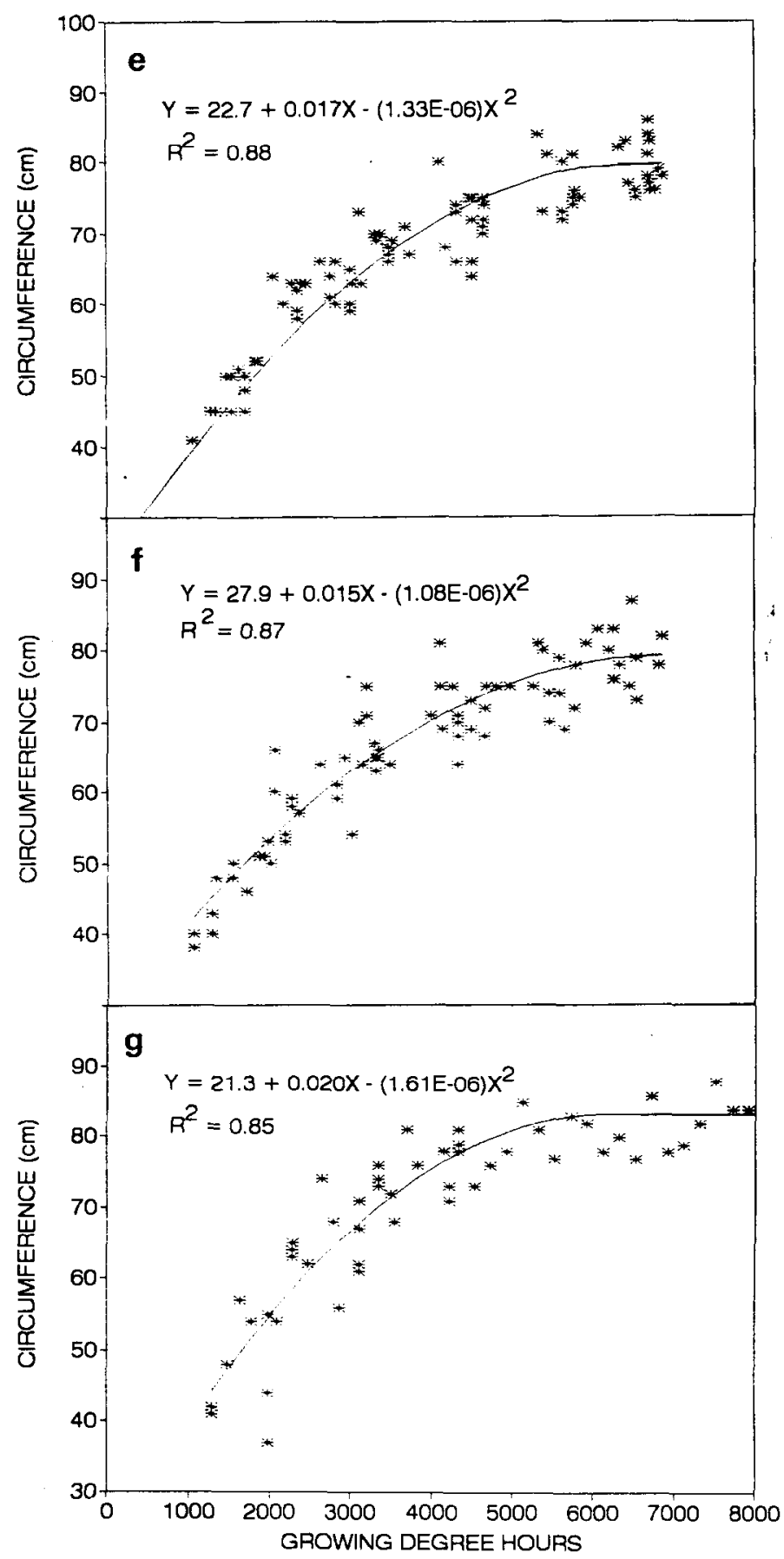

Fig. 2. The correlation between accumulated growing degree hours and 'Crimson Sweet' watermelon fruit enlargement (circumference) under different treatments during 198891 ( $\mathrm{a}=$ bare; ground; $\mathrm{b}=$ black plastic mulch; $\mathrm{C}=$ black plus clear plastic mulch; $\mathrm{d}=$ spunbonded polypropylene polyamide rowcover; $\mathrm{e}=$ spunbonded polyester fabric rowcover; $\mathrm{f}=$ perforated clear plastic rowcover and $\mathrm{g}=$ clear plastic mulch). All correlations werehighly significan $(\alpha=0.0000)$. 
Total yield was greatest under the PCP rowcover at Greenville and the SB-PF rowcover at Kaysville. Low yields from control plants at Greenville in 1991 contributed to yield differences between the control and other treatments. Fruit yields under PCP, SB-PF, SB-PP, CPM, BPM + CPM, and BPM were increased by $57 \%, 53 \%, 46 \%, 51 \%, 49 \%$, and $44 \%$, respectively at Logan. Yields for most treatments were generally greater in Kaysville than in Greenville mainly due to warmer temperatures and a longer growing season. Mean yield of control plants at Kaysville was $41 \%$ greater than at Logan. All mulches and rowcovers resulted in higher yields than on bare ground at Kaysville. Total yield under PCP, SB-PF and SB-PP rowcovers increased 35\%, $45 \%$, and $31 \%$, respectively.

The asymmetrical curvilinear model indicated a consistent heat unit requirement for Crimson Sweet watermelon plants to reach first male bloom, first female bloom, first fruit set and first harvest in uncovered plants or plants under rowcovers (Table 8). However, bare ground plants required considerably higher GDH to reach first male bloom, first female bloom, first fruit set than all other treatments mainly because bare soil was considerably cooler. Bare ground treatment required an average of 1000 more GDH than black plastic mulch to reach first male bloom.

Bloom date variability in watermelons contributes to the difficulty of predicting harvest dates; however,' the model still predicted the date of first harvest within 2 to 5 days under most treatments during different years. There was a high correlation between GDH and leaf numbers, leaf area, leaf dry weight and stem dry weight in uncovered as well as covered plants during early stages of growth (Table 9). The best correlation between GDH accumulation and leaf numbers, leaf dry weight and stem dry weight occurred under rowcover treatments (SB-PP, SB-PF, and $\mathrm{PCP}$ ). Leaf numbers, leaf dry weight and stem dry weights were generally the best correlated parameters with the GDH, but even leaf area and GDH had $r^{2}$ of 0.70 to 0.86 under different treatments. The bare ground leaf area was best correlated with GDH $\left(r^{2}=0.86\right)$ presumably because there was less mutual shading during the early stages of growth when the measurements were taken. The lowest correlation between leaf area and GDH was under PCP rowcovers $\left(r^{2}=0.70\right)$. The poorest overall correlations $\left(r^{2}\right.$ of 0.71 to 0.89$)$ were under BPM + CPM, mainly due to the high variability in soil temperature.

There was a nonlinear relationship between GDH accumulation and fruit enlargement (circumference) for all treatments (Fig. 2). Fruit enlargement was more closely related to GDH at the early stages of development. The response was linear until about 6000 GDH and became flat thereafter. The best correlations between GDH and fruit enlargement occurred on bare ground, BPM, SB-PF and PCP treatments ( $r^{2}$ values of 0.87 to 0.93$)$. Circumference enlargement under BPM + CPM was affected by the variability in soil temperatures under this treatment.

The proposed asymmetrical curvelinear model (Fig. 1) can be used to predict phenological stages of Crimson Sweet watermelon development as well as other growth parameters. The usable accuracy may extend over a rather wide range of climatic conditions. The correlations provided by the model may help growers in planning for cultural practices, and for allocating labor and machinery.

Growth analysis of sampled watermelon plants during early stages of development under various treatments was predictive of early yield. Growth analysis was indicative of growth responses of watermelon plants as effected by temperatures under rowcovers. Growth analysis could help determine proper timing to remove the rowcovers to minimize the negative effects of excessive heat generated under the rowcovers.

\section{Literature Cited}

Albright, L.D., D. Wolfe, and S. Novak. 1989. Modeling rowcover effects on microclimate and yield. II. Thermal model and simulations. J. Amer. Soc. Hort. Sci. 114:569-578.

Anderson, J.L. and E.A. Richardson. 1982. Utilizing meteorological data for modeling crop and weed growth, p. 449-461. In: J.L. Hatfield and I.J. Thomason (eds.). Biometeorology in integrated pest management. Academic Press, New York.

Buttrose, M.S. and M. Sedgley. 1978. Some effects of light intensity, daylength and temperature on growth of fruiting and non-fruiting watermelon. Ann. Bot. 42:599-608.

Gardner, G.P., R.B. Pearce, and R.L. Mitchell. 1985. Physiology of crop plants. Iowa State Univ. Press, Ames.

Hemphill, D.D., Jr., and G.D. Crabtree. 1988. Growth response and weed control in slicing cucumbers under rowcovers. J. Amer. Soc. Hort. Sci. 113:41-45.

Hemphill, D.D., Jr., and N.S. Mansour. 1986. Response of muskmelon to three floating rowcovers. J. Amer. Soc. Hort. Sci. 111:513-517.

Leopold, A.C. 1964. Plant growth and development. McGraw Hill, New York.

Levitt, J. 1980. Chilling, freezing, and high temperature stresses. 2nd ed. vol 1. Academic Press, New York.

Lombard, P. and E.A. Richardson. 1979. Physical principles involved in controlling phenological development, p. 429-440. In: B.J. Barfield and J.N. Moore (eds.). Modification of the aerial environment of crops. Amer. Soc. Agr. Eng.

Lorenz, O.A. and D.N. Maynard. 1988. Knott's handbook for vegetable growers. 3rd ed. Wiley, New York.

Mansour, N.S. 1989. Floating rowcovers on vegetable crops. The Grower (April):47-50.

Mat-r, C., W.J. Lamont, Jr., and M. Allison. 1991. Rowcovers improve seedless watermelon yields in an intensive vegetable production system. HortTechnology. 1:103-104.

Miller, G.A. 1989 Effect of rowcovers on harvest date of watermelon. Proc. Natl. Agr. Plastics Congr. 21:123-125.

Peirce L.C. and M.L. Crispi. 1989. Relationship between flowering and ripening dates modified in tomatoes by polyethylene mulch and rowcovers. HortScience 24:781-782.

Richardson, E.A. and S.G. Leonard. 1981. Climatic modeling of winter range lands in Utah, p. 210. 15th Conf. Agr. For. Meteorol., 1-3 Apr., Anaheim, Calif.

Sedgley, M. and M.S. Buttrose. 1978. Some effects of light intensity, daylength and temperature on flowering and pollen tube growth in the watermelon. Ann. Bot. 42:609-616.

Wang, J.Y. 1960. A critique of the heat unit approach to plant response studies. Ecology 41:785-790.

Wells, O.S. and J.B. Loy. 1985. Intensive vegetable production with rowcovers. HortScience 20:822-826.

Went, F.W. 1945. Plant growth under controlled conditions. The relationship between age, light, variety and thermoperiodicity of tomatoes. Amer. J. Bot. 32:469-479.

Wilson, M.A., P. Molahlane, V. Khan, and C. Stevens. 1987. Muskmelons on rowcovers and black plastic mulch. Proc. Natl. Plastics Congr. 21:123-125.

Wolfe, D.W., L.D. Albright, and J. Wyland. 1989. Modeling rowcover effects on microclimate and yield. I. Growth response of tomato and cucumber. J. Amer. Soc. Hort. Sci. 114:562-568. 